\title{
Finite Difference-Based Suboptimal Trajectory Planning of Biped Robot with Continuous Dynamic Response
}

\author{
Hayder F. N. Al-Shuka, Burkhard J. Corves, Bram Vanderborght, and Wen-Hong Zhu
}

\begin{abstract}
One of the clear problems encountered in the dynamic response of the biped robot is the discontinuity of the actuating torques/ground reaction forces at the transition instances during transferring form single support phase to double support phase and vice versa. Therefore, this paper suggests the linear transition function used in the biomechanics field for estimating the ground reaction forces during the double support phase such that gradual increase/decrease of the ground reaction forces can occur. The closure loop of the biped robot at the transition instances during DSP can be broken using the mentioned strategy. Consequently, the continuous dynamic response can be achieved. Two cases are simulated using the optimal control theory. The inverse dynamics-based optimization is preferred as a direct suboptimal tool because it can show less computation and easinessthan other optimal control approaches. Due to easiness of the finite difference approach, it is used for discretization of the dynamic equations and the imposed constraints to convert the dynamic optimal control problem into parameter optimization. The simulated case 1 have been used repeatedly in the literature, whereas the case 2 adopts the linear transition function of the ground reaction forces keeping the same generalized coordinates of the biped configuration at the transition instances. The results show the superiority of the suggested method to guarantee continuous actuating torques/ground reaction forces at the transition instances.
\end{abstract}

Index Terms-Biped robot, finite difference, optimization, suboptimal control.

\section{INTRODUCTION}

One of the important issues of the biped locomotion is the generation of the desired paths that ensure stability and avoid collision with obstacles [1]. Numerous approaches have been used to generate the motion of the biped robot as detailed in [2]. However, there are two efficient methods used for this purpose: center of gravity (COG)-based gait and the optimization-based gait. The former deals with a simplified model assuming all the masses of the biped robot are concentrated in the COG of the biped and there is pushing force at the ankle support foot without ankle torque applied

Manuscript received April16, 2013; revised July 2, 2013. This work was supported in part by a grant from German Academic Exchange Service (DAAD) and the Ministry of Higher Education and Scientific Research of Iraq (MoHESR).

Hayder F. N. Al-Shuka is with Department of Mechanism and Machine Dynamics, RWTH Aachen University, Aachen, Germany (e-mail: hayder.al-shuka@rwth-aachen.de).

B. J. Corves is with Department of Mechanism and Machine Dynamics, RWTH Aachen University, Aachen, Germany (e-mail: corves@igm.rwth-aachen.de ).

B. Vanderborght is with Department of Mechanical Engineering, VrijieUniversiteitBrussel, Brussel, Belgium (e-mail: bram.vanderborght@vub.ac.be).

Wen-Hong Zhu is with the Canadian Space Agency, Canada (e-mail:Wen-Hong.Zhu@asc-csa.gc.ca).
[3]. This method can guarantee the stability of the biped robot provided that full correction for the deviation of the zero-moment point is performed. However, it does not deal with the minimum energy, optimal design, and the different kinematic and dynamic constraints of the biped robot. The latter can be dealt successfully by the optimal control theory [4]. In general, the optimal control can be classified as: dynamic programming, indirect methods and direct methods as shown in Fig. 1. For details on advantages and disadvantages of the referred methods we refer to [5].

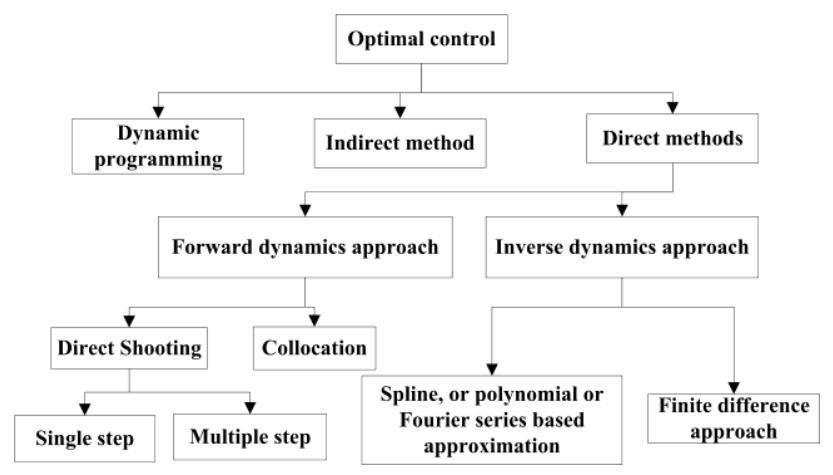

Fig. 1. The classification of the optimal control methods.

The direct methods of the optimal control are flexible methods which include transcribing the infinite dimensionproblem into finite-dimensional nonlinear programming (static or parameter optimization). This can be implemented by discretization of the controls and/or the states, depending on the selected discretization approach, and solving the problem using one of the nonlinear programming algorithms such as sequential quadratic programming (SQP), interior points, genetic algorithm (GA) etc. Although its ease and robustness, this method can only give suboptimal/approximate solution [6]-[9]. One of the preferred methods of the direct approaches is the inverse dynamics- based optimization which has three distinctive features: (a) it does not need the inverse mass matrix, (b) only the states of the target system are discretized, and (c)the ability to convert the original optimal control into algebraic equations which are easy to deal with. Thus, this method can show less computation than other optimal control approaches. The discritization task can be performed using either the polynomials (splines) or the finite difference approach. Due to easiness of the latter, it is used for discretization of the dynamic equations and the imposed constraints to convert the dynamic optimal control problem into parameter optimization.

One of the problems encountered in the analysis of the trajectory planning and control of the biped robot is the 
discontinuity of the actuating torques/ground reaction forces at the transition instances (from single support phase SSP to double support phase DSP and vice versa, briefly, SSP/DSP/SSP). This problem occurs due to the varying configuration of the biped robot from open chain mechanism to closed chain mechanism and vice versa. Via investigating the literature [10]-[14], this problem can still exist despite of the assumption of equal states (angular displacement, velocity and acceleration) at the transition instances. Therefore, this paper focuses on the solution of the discontinuity of the actuating torques/ground reaction forces guaranteeing continuous states and using the suboptimal trajectory planning.

The structure of the paper is as follows. Problem statement is introduced in Section II. Section III investigates the methods and materials used. While Section IV shows the simulation results and discussions. The conclusion is considered in Section V.

\section{Problem Statement}

The complete gait cycle of human walking consists of two main successive phases: the single support phase (SSP) and the double support phase (DSP) with intermediate sub-phases [15]-[17]. The DSP arises when both feet contact the ground resulting in a closed chain mechanism. While the SSP starts when the rear foot is not supported by the ground with front foot flat on the ground. One should note that the percentage of the DSP is about $20 \%$ during one stride of the gait cycle, while the SSP is about 80\%[17], [18]. Because of the shortness of this phase, the forward velocity of the COG could be high. In additions the biped robot behaves as closed chain mechanism (over-actuated mechanism) during DSP with infinity combinations of actuating torques/ground reaction forces. There are miscellaneous walking patterns, however we propose the walking pattern referred by Fig. 2c because it has a simple configuration during the DSP. In this walking pattern, the DSP consists of rotation of the front foot about the heel until it will be flat on the ground, while the rare foot rotates about its tip until it will toe off. The following dynamic analysis is used by the literature [10]-[13].

During the SSP, the biped robot behaves as an open-chain mechanism, therefore, the governed Lagrangian dynamic equation can be written as

$$
M \ddot{q}+C \dot{q}+g=A \tau, q=\left[q_{2}, \ldots, q_{7}\right]
$$

where $M \in \mathbb{R}^{n_{q} \times n_{q}}$ is the mass robot matrix, $n_{q}$ denotes the number of the generalized coordinates which is equal to the degrees of freedom DOFs $\left(n_{q}=n=n_{\tau}=6\right), n$ and $n_{\tau}$ represent the number of DOFs and the number of actuators respectively, $q, \dot{q}$ and $\ddot{q} \in \mathbb{R}^{n_{q}}$ are the absolute angular displacement, velocity and acceleration of the robot links, $C \in \mathbb{R}^{n_{q} \times n_{q}}$ represents the Coriolis and centripetal robot matrix, $g \in \mathbb{R}^{n_{q}}$ is the gravity vector, $A \in \mathbb{R}^{n_{q} \times n_{\tau}}$ is a mapping matrix derived by the principle of the virtual work [19], [20], and $\boldsymbol{\tau} \in \mathbb{R}^{n_{\tau}}$ is the actuating torque vector.

During DSP, the configuration of the biped mechanism changes, therefore, the constrained Lagrangian dynamic equation during this phase is

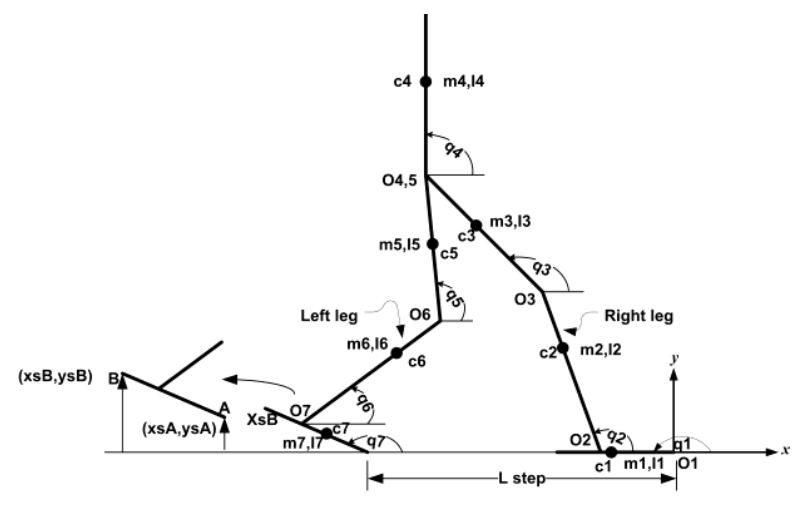

(a)

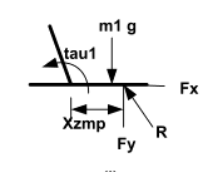

(i)

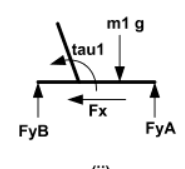

(ii)
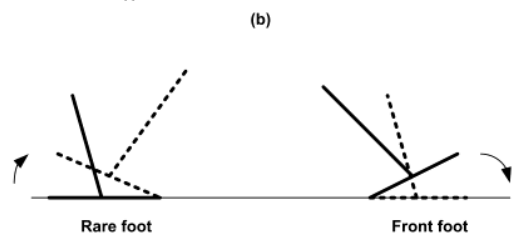

(c)

$$
M \ddot{q}+C \dot{q}+g=A \tau+J^{T} \lambda, q=\left[q_{1}, \ldots, q_{7}\right]
$$

Fig. 2. Biped robot structure.

$$
\varphi(q)=0
$$

where $\varphi($.$) represents the constraint equation, n_{q}=7, n=$ 5 and $n_{\tau}=6$ with the same notations mentioned earlier. $J \in \mathbb{R}^{2 \times n_{q}}$ represents the jacobian matrix resulting from the constrained motion of the biped robot, and $\lambda \in \mathbb{R}^{2}$ is the constrained force vector, i.e. the reaction forces of the left foot.

During this phase, the biped robot undergoes over-actuation because its DOFs reduce to 5 DOFs (3 DOFs for hip and 2 DOFs for the two feet). Consequently, there are two redundant actuating torques. As noted, the number of generalized coordinates $n_{q}$ of the biped changes from 6 to 7 and consequently the mapping matrix of the actuating torques changes also. The well-known solution of the over-actuated dynamic equation of the DSP is as follows

$$
M \ddot{q}+C \dot{q}+g=\left[\begin{array}{ll}
A & J^{T}
\end{array}\right]\left[\begin{array}{l}
\tau \\
\lambda
\end{array}\right]
$$

One of the feasible solutions can be found using the Pseudo-inverse matrix as described in (5).

$$
\left[\begin{array}{l}
\tau \\
\lambda
\end{array}\right]=\left[\begin{array}{ll}
A & J^{T}
\end{array}\right]^{\#}(M \ddot{q}+C \dot{q}+g)
$$

where [ ] ${ }^{\#}$ refers to the Pseudo-inverse of the target matrix.

The following points should be noted:

1) This method cannot guarantee the smooth transition and continuities of the actuating torques/ground reaction forces at the transition instances (SSP/DSP/SSP) as we will see in later sections.

2) From equation (2), it is noted even though the assumption of equal states at the transition instances, the 
discontinuity can occur because the structure of the mapping matrix changes during this phase.

3) It is known that the optimization tool can implement what the analyst provides with the required constraints. Consequently, (3) needs other constraints as follows.

$$
\begin{aligned}
& J \dot{q}=0 \\
& \dot{J} \dot{q}+J \ddot{q}=0
\end{aligned}
$$

However, these constraints can result in infeasible solutions. Therefore, the literature has used (3) only. As a result, application of the dynamic equation (2) during DSP from the left foot and go around the tip of the other foot may not give the same results if we apply the dynamic equation from the opposite side. Consequently, the most deviation of the discontinuity can result in at the end of DSP.

\section{METHODS}

This section will describe two selected case used for dealing with (2). Moreover, the dynamic modeling, the constraints and the optimal trajectory planning of 7-link biped robot during complete gait cycle will be introduced.

\section{A. Dynamic Modeling \\ 1) Case 1}

This is the exactly same case presented in the previous section and has been used in the literature. We simulated this case for comparative purposes. The dynamic equations during the SSP and the DSP can be described through (1)-(5).

\section{2) Case 2}

Since the biped robot does not have a unique solution during the DSP, we assume a linear transition function for the ground reaction forces of the front foot as follows [21]-[24].

$$
\lambda=\left(\frac{t-t_{s}}{t_{d}-t_{s}}\right) m_{\operatorname{cog}}\left(\ddot{r}_{\operatorname{cog}}+[0, g]\right)
$$

where $\ddot{r}_{\text {cog }}=\left[\begin{array}{ll}\ddot{x}_{\operatorname{cog}} & \ddot{y}_{\text {cog }}\end{array}\right]^{T}, t_{s}, t_{d}$ denote the time of the SSP and DSP respectively, $m_{c o g}$ is the mass of the center of gravity and $\ddot{r}_{\text {cog }}$ represents the acceleration of the biped COG. Whereas the rare foot has the following ground reaction forces

$$
f=m_{\operatorname{cog}}\left(\ddot{r}_{\operatorname{cog}}+[0, g]\right)-\lambda
$$

Thus, the actuating torques can be found as follows.

$$
\left[\begin{array}{l}
\tau \\
\lambda
\end{array}\right]=[A]^{\#}\left(M \ddot{q}+C \dot{q}+g-J^{T} \lambda\right)
$$

In effect, at the initial time of DSP, we can note, according to (7), $\lambda=0$. Therefore, the closed chain mechanism is broken and (1) can be used successfully with the same generalized coordinates used in SSP. At the end of the DSP, the ground reaction forces at the front foot can take the full value of the inertial COG forces, i.e. the linear transition function is equal to 1 , and according to (8), $f=0$. As a result there is no need to use the closed chain equation at the end conditions of the DSP. The continuity conditions of the actuating torques/ground reaction forces can be explicitly satisfied.

\section{B. Kinematic and Dynamic Constraints}

DuringSSP $t_{0} \leq t \leq t_{s}$, where $t_{0}$ is the initial time. Initial configuration of the swing leg:

$$
\begin{gathered}
x_{S A}\left(t_{0}\right)+L_{\text {step }}=0, y_{S A}\left(t_{0}\right)=0 \\
\dot{x}_{S A}\left(t_{0}\right)=0, \dot{y}_{S A}\left(t_{0}\right)=0
\end{gathered}
$$

Final configuration of the swing leg:

$$
\begin{aligned}
x_{s B}\left(t_{s}\right)-L_{\text {step }} & =0, y_{s B}\left(t_{s}\right)=0 \\
\dot{x}_{s B}\left(t_{s}\right) & =0, \dot{y}_{s B}\left(t_{s}\right)=0
\end{aligned}
$$

where all the notations are shown in Fig.1.

Hip motion:

$$
\begin{gathered}
x_{\text {hip }}\left(t_{0}\right)=-1.4 \\
x_{\text {hip }}\left(t_{s}\right)=1.4 \\
y_{\text {hip }}\left(t_{0}\right)=y_{\text {hip }}\left(t_{s}\right)=0.8881 \\
\dot{x}_{\text {hip }}(t)>0 \\
h_{\text {min }} \leq y_{\text {hip }}(t)
\end{gathered}
$$

In effect, (12)-(14) are optional for guaranteeing natural motion. It is assumed that the hip of the biped robot can move with displacement of $0.7 \times L_{\text {step }}$ during the SSP and $0.3 \times L_{\text {step }}$ during the DSP. Where $L_{\text {step }}$ represents the length of walking step which is taken as 0.4 [m]. This assumption coincides approximately with the natural motion of human gait.

Swing foot motion:

$$
\begin{aligned}
& 0<y_{S A}(t) \\
& 0<y_{S B}(t)
\end{aligned}
$$

Relative displacement of the knee joints and swing ankle:

$$
\begin{aligned}
& 5^{0} \leq q_{3}(t)-q_{2}(t) \leq 90^{0} \\
& 5^{0} \leq q_{5}(t)-q_{6}(t) \leq 90^{0} \\
& 90^{0} \leq q_{7}(t)-q_{6}(t) \leq 270^{0}
\end{aligned}
$$

ZMP-constraint:

In general, there are two concepts used in the literature as follows:

Concept.1: This is commonly used in the field of biped robot which states that the location of reaction force is equal to the ZMP as long as the biped mechanism is stable, as shown in Fig. 2b, therefore;

$$
\begin{gathered}
\tau_{1}(t)+F_{y}(t) x_{Z M P}(t)-m_{1} g d_{1}=0 \\
x_{Z M P}(t)=\left(m_{1} g d_{1}-\tau_{1}(t)\right) / F_{y}(t)
\end{gathered}
$$

Thus, the necessary associated constraint is

$$
-\left(l_{f 2}+l_{f 1}\right) \leq x_{Z M P}(t) \leq 0
$$

where $d_{1}=\overline{O_{1} c_{1}}, l_{f 1}=\overline{O_{1} A}$ and $l_{f 2}=\overline{O_{1} B}$

In addition, the following constraints of the ground reaction forces should be satisfied 


$$
\begin{gathered}
-F_{y}(t)<0 \\
-F_{x}-\mu F_{y}(t)<0 \\
F_{x}-\mu F_{y}(t)<0
\end{gathered}
$$

Concept.2: [25], [26]. It is assumed that the ground reaction forces can be equivalently represented by two normal forces $F_{y A}, F_{y B}$ applied at end points $\mathrm{A}$ and $\mathrm{B}$ of the foot, with a horizontal force acting in the sole, as shown in Fig. 2b (ii). Thus, the conditions that should be satisfied are

$$
\begin{gathered}
-F_{y A}(t)<0 \\
-F_{y B}(t)<0 \\
-F_{x}(t)-\mu\left(F_{y A}(t)+F_{y B}(t)\right)<0 \\
F_{x}(t)-\mu\left(F_{y A}(t)+F_{y B}(t)\right)<0
\end{gathered}
$$

Bounded constraints

$$
\begin{gathered}
q_{\min } \leq q(t) \leq q_{\max } \\
\dot{q}_{\min } \leq \dot{q}(t) \leq \dot{q}_{\max }
\end{gathered}
$$

During $\operatorname{DSP}\left(t_{s}<t<t_{d}\right)$

$$
\varphi(q)=0
$$

The same equation as (21) for the rare and front feet.

$$
\dot{x}_{\text {hip }}(t)>0 \text {. }
$$

The same equations as (18) and (23).

Remark:We do not impose constraints on the stability criterion during this dynamically stable phase (DSP). However, the ZMP can be obtained from the center of the pressure for the contact feet as follows [27]:

$$
\operatorname{cop}=\operatorname{cop}_{f} \frac{f_{y f}}{f_{y f}+f_{y r}}+\operatorname{cop}_{r} \frac{f_{y r}}{f_{y f}+f_{y r}}
$$

where $c o p, c o p_{f}$ and $c o p_{r}$ represent the center of pressure for the biped robot during DSP, the front foot cop and the rare foot cop respectively, $f_{y f}$ and $f_{y r}$ are the normal component of the ground reaction forces for the front and rear foot respectively.

\section{Finite Difference-Based Parameter Optimization}

With the finite-difference approach, the angular displacements of the dynamic system are segmented, whereas the angular velocities and accelerations of the dynamic system can be approximated as follows.

$$
\begin{gathered}
\dot{q}\left(t_{k}\right)=\left(q\left(t_{k+1}\right)-q\left(t_{k-1}\right)\right) / 2 . \Delta t, k=0, \ldots, N \\
\ddot{q}\left(t_{k}\right)=\left(q\left(t_{k+1}\right)-2 q\left(t_{k}\right)+q\left(t_{k-1}\right)\right) / \Delta t^{2}, k=0, \ldots, N
\end{gathered}
$$

where $N$ represents the number of segments (intervals). For detail we refer to [28], [29]. The formulations of the finite difference-based optimization will be described separately for SSP and DSP for better comprehension.

\section{During SSP}

$$
\text { Determin: } Y=\left[q\left(t_{0}\right), \ldots, q\left(t_{N_{1}}\right), \dot{q}\left(t_{0}\right), \dot{q}\left(t_{N_{1}}\right)\right]
$$

Minimize:

$$
J=\int_{t 0}^{t s} \tau^{T} \tau d t=\sum_{k=0}^{N_{1}-1} \tau_{k}^{T} \tau_{k} \cdot \Delta t
$$

Subject to:

$$
\begin{gathered}
M\left(q\left(t_{k}\right)\right) \ddot{q}\left(t_{k}\right)+C\left(q\left(t_{k}\right), \dot{q}\left(t_{k}\right)\right) \dot{q}\left(t_{k}\right)+g\left(q\left(t_{k}\right)\right)= \\
A \tau\left(t_{k}\right) \quad, k=0, \ldots, N_{1}
\end{gathered}
$$

Moreover, the kinematic and dynamic constraints (10)-(23) can be written in general form as follows:

$$
\begin{gathered}
a_{1}\left(q\left(t_{0}\right), \tau\left(t_{0}\right), t_{0}\right) \leq 0 \\
a_{2}\left(q\left(t_{0}\right), \tau\left(t_{0}\right), t_{0}\right)=0 \\
b_{1}\left(q\left(t_{N_{1}}\right), \tau\left(t_{N_{1}}\right), t_{N_{1}}\right) \leq 0 \\
b_{2}\left(q\left(t_{N_{1}}\right), \tau\left(t_{N_{1}}\right), t_{N_{1}}\right)=0 \\
c_{1}\left(q\left(t_{k}\right), \tau\left(t_{k}\right), t_{k}\right) \leq 0, \quad k=0, \ldots, N_{1} \\
c_{2}\left(q\left(t_{k}\right), \tau\left(t_{k}\right), t_{k}\right)=0, \quad k=0, \ldots, N_{1} \\
q_{l} \leq q\left(t_{k}\right) \leq q_{u}, \quad k=0, \ldots, N_{1}
\end{gathered}
$$

where $N_{1}$ denotes the number of segments used for discritezation of SSP, $c_{0}$ and $L$ are scalar functions of the indicated arguments, $J$ is a scalar performance index, $a_{1}$ and $a_{2}$ are the initial constraints, $b_{1}$ and $b_{2}$ are the final constraints, $\boldsymbol{c}_{\mathbf{1}}$ and $\boldsymbol{c}_{\mathbf{2}}$ are the path constrains and (33) refers to the bound constraints of the input control and the states.

\section{During DSP}

For the simulated cases 1 and 2, the formulations of the finite difference-based optimization can be described as follows:

Determine:

Minimize:

$$
Y=\left[q\left(t_{1}\right), \ldots, q\left(t_{N_{2}}\right)\right]
$$

$$
\begin{gathered}
J=\int_{t_{s}}^{t_{d}}\left(\tau^{T} \tau+\lambda^{T} \lambda\right) d t=\sum_{k=0}^{N_{2}-1} \tau_{k}^{T} \tau_{k} \cdot \Delta t+ \\
\sum_{k=0}^{N_{2}-1} \lambda_{k}^{T} \lambda_{k} \cdot \Delta t
\end{gathered}
$$

Subject to:

$$
\begin{gathered}
M\left(q\left(t_{k}\right)\right) \ddot{q}\left(t_{k}\right)+C\left(q\left(t_{k}\right), \dot{q}\left(t_{k}\right)\right) \dot{q}\left(t_{k}\right)+g\left(q\left(t_{k}\right)\right)= \\
A \tau\left(t_{k}\right)+J\left(t_{k}\right)^{T} \lambda\left(t_{k}\right), \quad k=1, . .
\end{gathered}
$$

In addition to the constraints denoted (30)-(33).

\section{Simulation Results AND Discussions}

Seven-link biped robot was investigated for simulation purpose. The physical parameters of the biped structure are shown in TABLE I. It is assumed that the time of the walking step $\left(T_{\text {step }}\right)$ is equal to $0.625(\mathrm{~s})$. The time of the SSP and DSP can be calculated as $0.8 \times T_{\text {step }}$ and $0.2 \times T_{\text {step }}$ respectively. Consequently, the velocity of the walking step is equal to $0.64(\mathrm{~m} / \mathrm{s})$. The finite difference approach was used to discretize the angular displacement of the biped and to transcribe the dynamic optimization problem into static optimization. The number of divisions used for SSP was 10. We tried to increase the number of divisions; however, the same results were obtained. Due to the small time of DSP, 6 divisions were used for discretization of the angular displacement. Increasing the number of divisions during this 
short phase could result in infeasible solution. Then, after discretization and formulating the imposed constraints, fmincon MATLAB routine was used successfully for solution of the parameter optimization.

Two simulated cases were investigated for dealing with the continuity of the actuator torques /ground reaction forces of the biped at the transition instances. As expected, using the strategy referred by case 1 , can lead to large discontinuity at the transition instances which can be explained by the three points referred by Section II. The large discontinuity could be obtained at the end of the DSP due to the third point mentioned in Section II. The states of the biped robot are known from the previous and next SSP; therefore, it cannot be imposed additional constraints on the actuating torques/ground reaction forces at the transition instances. Figures 3 and 4 show the actuating torques and the ground reaction forces during the three walking phases (SSP/DSP/SSP). It should be noted that the normal component of the ground reaction forces could be negative in sign at the transition instances, which is unrealistic, because the actuating torques/reaction forces at the transition phases are obtained from the known states at the end conditions. Consequently, we cannot impose constraints on the reaction forces or any constraints at the end conditions. In effect, it is possible to get continuous actuating torques/reaction forces if we release the velocity and acceleration at the end conditions of the DSP. So, constraints could be imposed on the actuating torques/reaction forces and the acceleration to be continuous at the expense of discontinuous angular velocity. However, we cannot get complete continuous states. Therefore, the simulated case 2 could be better solution.

Using the linear transition function for the ground reaction forces during DSP in the simulated case 2 can guarantee the continuity of the actuating torques/ground reaction forces at the transition instances due to the smooth gradual increase/decrease of the ground reaction forces at the front/rare feet. The objective function is better than case 1 as referred such that it reduces from $110758.21\left(N^{2} . \mathrm{m}^{2} . \mathrm{s}\right)$ at case 1 to $1695.2\left(N^{2} \cdot m^{2} \cdot s\right)$ at case 2. Figs. 5-8 show the actuating torques, ground reaction forces, cop and the stick diagram for case 2 . In effect, the same stick diagram can be got during the two mentioned cases.

Remark:Form Fig. 5, the value of one of the ankle torque is above 100 (N. m) which is not preferred with regard to the stability problem. This occurs because we do not impose bounded constraints on the actuator torques.

Remark:We do not impose a constraint on the torso configuration to be vertical because this can increase the energy consumption and complicate the optimization problem. Instead, bounded limits are used for the torso vertical configuration, which are easy to deal in fmincon MATLAB routine.

\begin{tabular}{ccccc}
\multicolumn{5}{c}{ TABLE I: THE PHYSICALPARAMETERS OF THE BIPED ROBOT } \\
\hline \hline$i$ & $l_{i}$ & $d_{i}=\overline{O_{i} c_{i}}$ & $m_{i}$ & $I_{i}$ \\
\hline 1 & 0.3 & 0.126 & 2.05 & 0.016 \\
2 & 0.45 & 0.26 & 3.61 & 0.06 \\
3 & 0.45 & 0.261 & 3.69 & 0.062 \\
4 & 0.45 & 0.2 & 10.3 & 0.145 \\
5 & 0.45 & 0.189 & 3.66 & 0.06 \\
6 & 0.45 & 0.192 & 3.53 & 0.058 \\
7 & 0.3 & 0.073 & 2.05 & 0.016 \\
\hline \hline
\end{tabular}

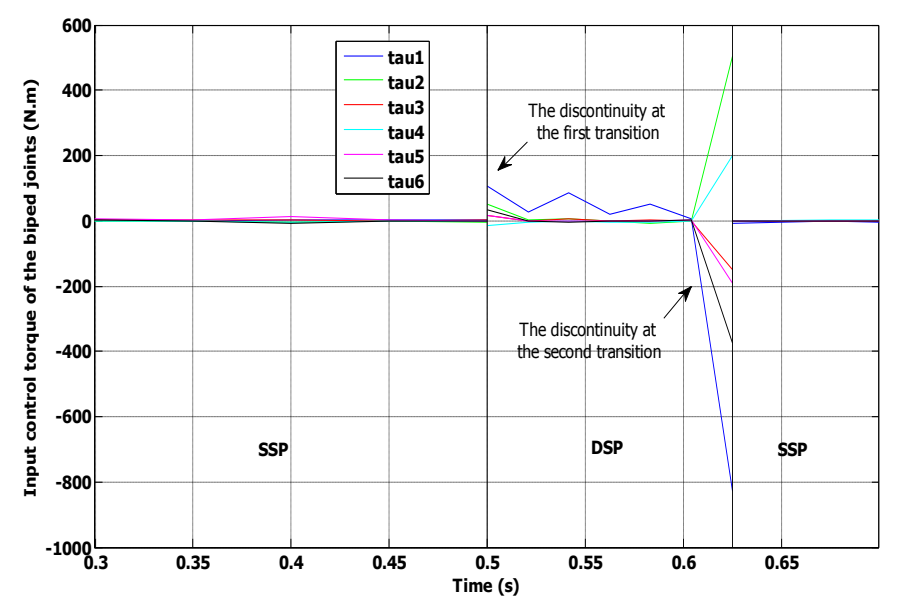

Fig. 3. Actuating joint torques at case 1.

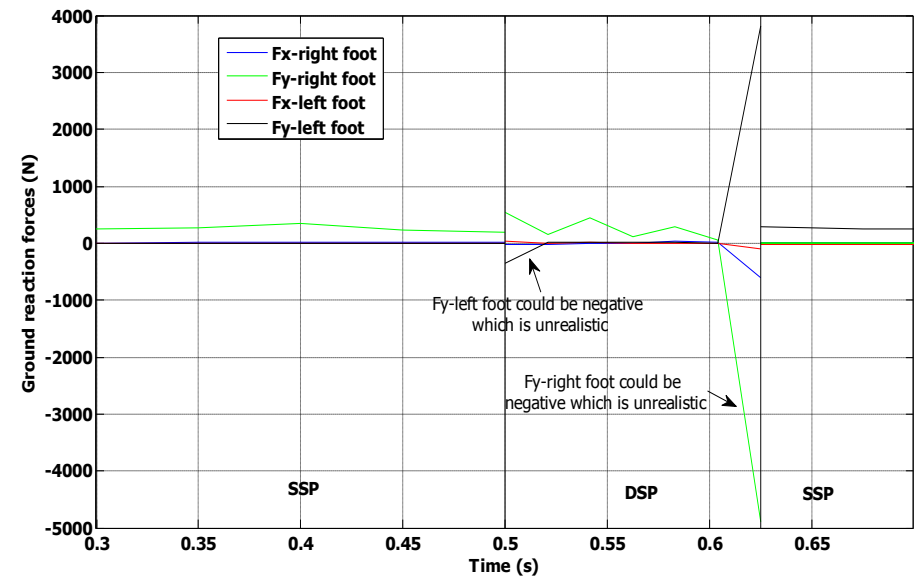

Fig. 4. Ground reaction forces at case 1.

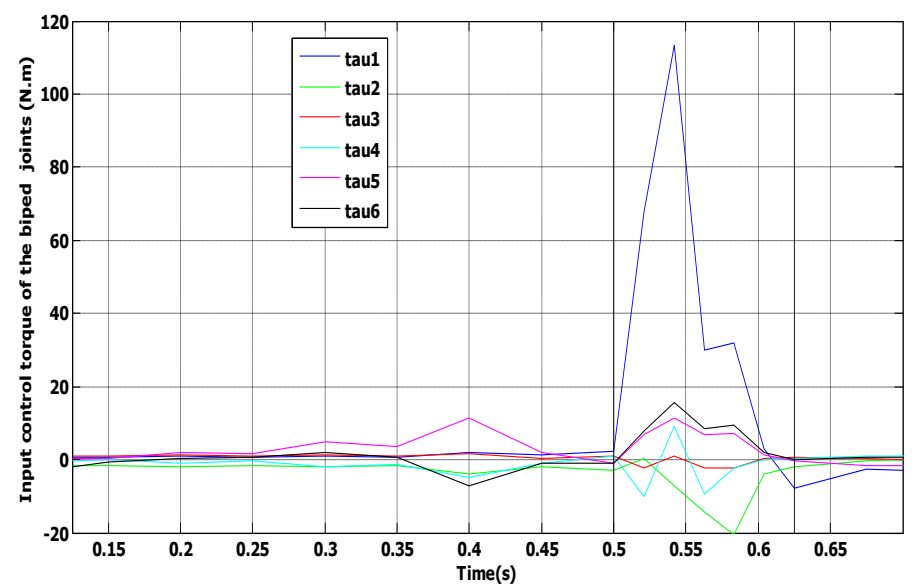

Fig. 5. Actuating joint torques at case 2 .

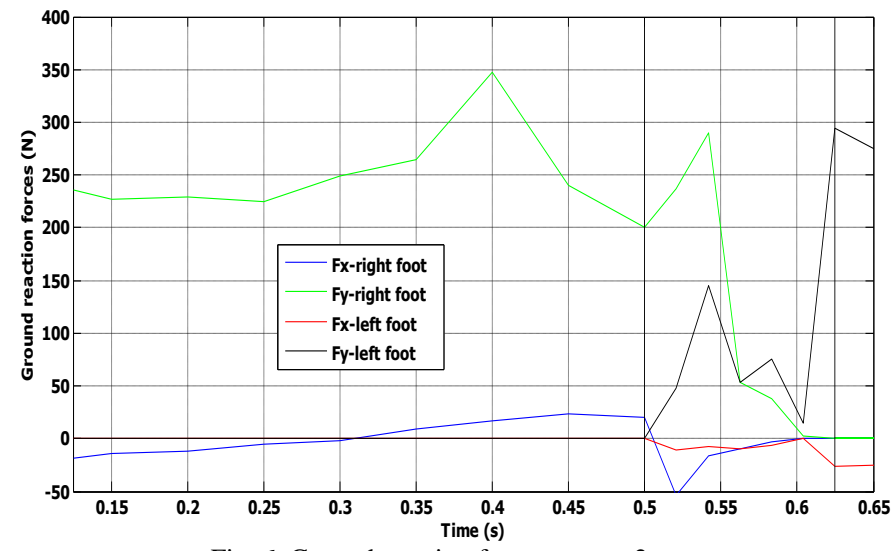

Fig. 6. Ground reaction forces at case 2. 


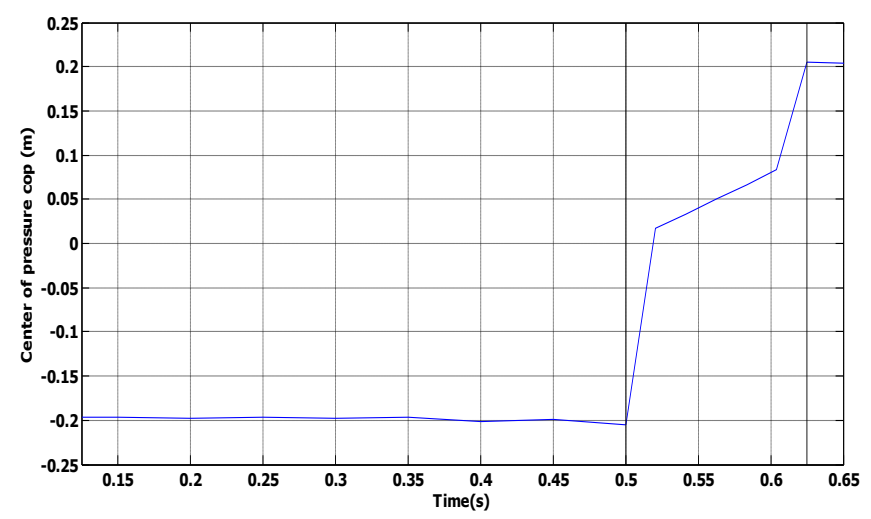

Fig. 7. Center of pressure at case 2 .

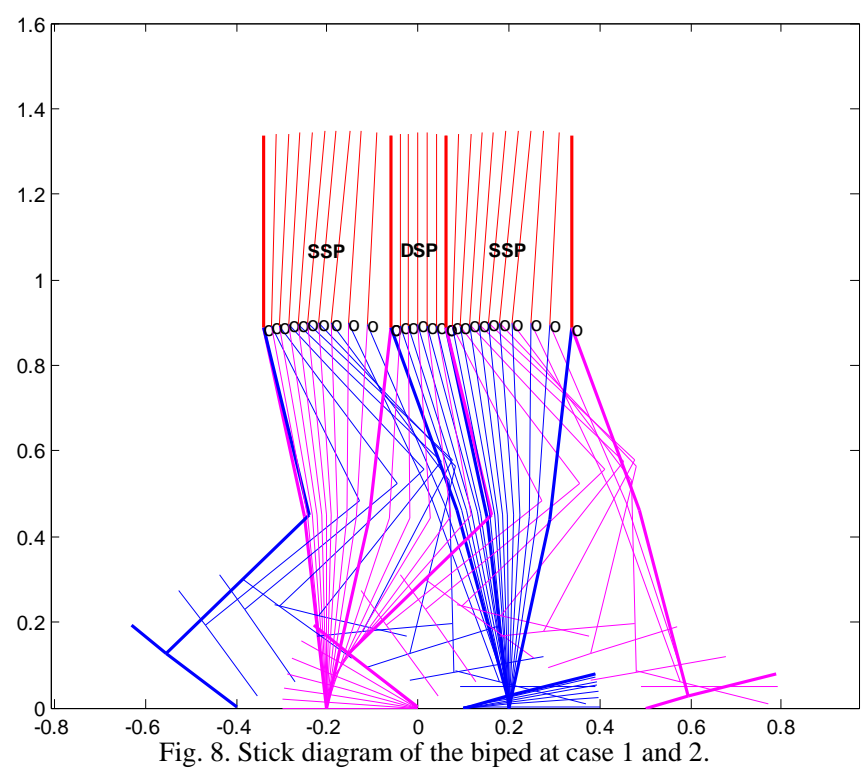

\section{CONCLusions}

This paper deals with the problem of the discontinuous actuating torques/reaction forces at the transition instances using the linear shift function for the ground reaction forces This strategy can guarantee the continuity of the actuating torques/reaction forces and the states of the biped robot.

This study deals with steady gait cycle rather than accelerating and decelerating cycles in which the biped robot can move from rest and stop. Moreover, the effect of toe and/or heel joints on the walking parameters, nature of walking and the energy consumption are not considered. Using the recursive Newton Euler formulation can improve the computational complexity. Optimal control theory combined with the recursive Newton Euler formulation is necessary for complex robotic system such as biped robot. Therefore, further study is needed to deal with the above important points.

\section{REFERENCES}

[1] P. R. Vundavilli and D. K. Pratihar, "Gait planning of bipedrobots using soft computing: An attempt to incorporate intelligence," in Intelligent Autonomous Systems: Foundation and Applications, D. K. Pratihar and L. C. Jain, Eds. Germany: Springer-Verlag, 2010, ch. 4, pp. 57-85.

[2] H. F. N. Al-Shuka, F. Allmendinger, and B. Corves, "Modeling, stability and walking pattern generators of biped robots: A review," Unpublished.

[3] S. Kajita and K. Tani, "Experimental study of biped dynamic walking in the linear inverted pendulum mode," in Proc. IEEE Conf. Robotics and Automation, vol. 3, pp. 2885-2891, 1995.
[4] C. Chevallereau, G. Bessonnet, G. Abba, and Y. Aoustin, Bipedal Robots, Modeling , Design and Building Walking Robots, $1^{\text {st }}$ ed. ,U.K., John Wiley and Sons Inc., 2009,ch. 4, pp. 219-265.

[5] H. F. N. Al-Shuka, B. Corves, and W. H. Zhu, On the dynamic optimization of biped robot.

[6] M. G. Pandy, F. C. Anderson, and D. G. Hull, "A parameter optimization approach for the optimal control of large-scale musculoskeletal," J. Biomech. Eng., vol. 114, no. 4, pp. 450-460, 1992.

[7] M. Diehl, "Numerical optimal control," Optimization in Engineering Center and Electrical Engineering Department, K. V. Leuven, Belgium, 2011.

[8] D. G. Hull, "Conversion of optimal control problems into parameter optimization problems," AIAA, Guidance, Navigation and control performance Conference, July, 1996,San Diego, CA.

[9] C. J. Goh and K. L. Teo, "Control parameterization, A unified approach to optimal problem with general constraints," Automatica, vol. 24, no. 1, pp. 3-18, 1988.

[10] G. Bessonnet, P. Seguin, and P. Sardain, "A parametric optimization approach to walking pattern synthesis," The International Journal of Robotics Research, vol. 24, no. 7, pp. 523-536,2005.

[11] G. Bessonnet, S. Chesse, and P. Sardain, "Optimal gait synthesis of a seven-link planar biped," The International Journal of Robotics Research, vol. 23, no. 10-11, pp. 1059-1073, 2004.

[12] P. Seguin and G. Bessonnet, "Generating optimal walking cycles using spline-based state-parameterization," International Journal of Humanoid Robotics, vol. 2, no. 1, pp. 47-80, 2005.

[13] N. Jamshidi and M. Rostami, "Gait optimization of biped robot during double support phase by pure dynamic synthesis," American Journal of Applied Science, vol. 5, no. 9, pp. 1175-1181, 2008.

[14] V. Bram et al., "Overview of the Lucy project: Dynamic stabilization of a biped powered by pneumatic artificial muscles," Advanced Robotics, vol. 22, no.10, pp. 1027-1051, 2008.

[15] M. H. P. Dekker, "Zero-moment point method for stable biped walking," Internship report, Eindhoven, 2009.

[16] G. Ozyurt, "3-D Humanoid gait simulation using an optimal predictive control," MSc. Thesis, Middle East Technical University, 2005.

[17] J. Patton, "Gait Section, PartB, Kinesiology," Lecture notes, Department of physical therapy and human movement science, Northwestern University, Medical School, 2001.

[18] C. L.Golliday and H. Hemami, "An approach to analyzing biped locomotion dynamics and designing robot locomotion controls," IEEE Transactions on Automatic Control, vol. AC-22, no. 6, Dec. 1977.

[19] A. Hamon and Y. Aoustin, "Cross four-bar linkage for the knees of a planar bipedal robot," in Proc. IEEE-RAS International Conference on Humanoid Robots, 2010, pp. 379-384.

[20] S. Tzafestas, M. Raibert, and C. Tzafestas, "Robust sliding mode control applied to 5-link biped robot," Journal of Intelligent and Robotic Systems, vol. 15, pp. 67-133, 1996.

[21] M. Y. Zarrugh, "Kinematic prediction of intersegment loads and power at the joints of the leg in walking," J. Biomechanics, vol. 10, no. 10, pp. 713-725, 1981.

[22] B. Koopman, H. J. Grootenboer, and H. J. de Jonegh, "An inverse dynamics model for the analysis, reconstruction and prediction of bipedal walking," J. Biomechanics, vol. 28, no. 11, pp. 1369-1376, 1995.

[23] L. Ren, R. K. Jones, and D. Howard, "Whole body inverse dynamics over a complete gait cycle based only on measured kinematics," $J$. Biomechanics, vol. 41, pp. 2750-2759, 2008.

[24] A. G. Alba and T. Zielinska, "Postural equilibrium criteria concerning feet properties for biped robots," Journal of Automation, mobile robotics and Intelligent Systems, vol. 6, no. 1, pp. 22-27, 2012.

[25] M. Rostami and G. Bessonnet, "Sagittal gait of a biped robot during the single support phase. Part 2: Optimal motion," Robotica, vol. 19, pp. 241-253, 2001.

[26] T. Saidouni and G. Bessonnet, "Generating globally optimised sagittal gait of a biped robot," Robotica, vol. 21, pp. 199-210, 2003.

[27] C. Pop, A. Khajepour, J. P. Huissoon, and A. E. Patla, "Experimental/analytical analysis of human locomotion using bondgraphs," ASME, vol. 125, pp. 490-498, 2003.

[28] Q. Wang, "A study of alternative formulations for optimization of structural and mechanical systems subjected to static and dynamic loads," Ph.D. Dissertation, University of IOWA, USA, 2006.

[29] Q. Wang and J. S. Arora, "Alternative formulations for transient dynamic response optimization," AIAA, vol. 43, no. 10, pp. 2188-2195, 2005 .

Hayder F. N. Al-Shuka was born in Baghdad, Iraq, in 1979. He received the B.Sc and M.Sc. degrees from Baghdad and Al-Mustansiriya Universities respectively, Iraq, in 2003 and 2006 respectively. Since 2006, he has been appointed as an assistant lecturer in Baghdad University at the department of 
Mechanical Engineering. He is currently PhD student at the RWTH Aachen University at the Department of Mechanism and Machine Dynamics. His research interests include the walking patterns and control of biped robots.

Burkhard J. Corves was born in Kiel, Germany, in 1960. He received the Diploma and $\mathrm{PhD}$ degrees in Mechanical Engineering from RWTH Aachen University, Aachen, Germany, in 1984 and 1989 respectively. From 1991 until 2000, he got teaching assignment in RWTH Aachen University. Since 2000, he has been appointed as university professor and director of the department of Mechanism and Machine Dynamics of RWTH Aachen University. The research interests of Prof. Dr. Corves include the kinematics and dynamics of mechanisms and robots.

Bram Vanderborght received the degree in the study of Mechanical Engineering at the VrijeUniversiteitBrussel in 2003. In May 2007 he received his $\mathrm{PhD}$ in Applied Science. The focus of his research was the use of adaptable compliance of pneumatic artificial muscles in the dynamically balanced biped Lucy. In May-June 2006 he performed research on the humanoids robot HRP-2 at the Joint Japanese/French Robotics Laboratory (JRL) in AIST, Tsukuba (Japan). From October 2007-April 2010 he worked as post-doc researcher at IIT (Italy) on locomotion and compliant actuation. Since October 2009, he has been appointed as professor at the VUB. He is member of the Young Academy of the Royal Flemish Academy of Belgium for Science and the Arts. His research interests includes cognitive and physical human robot interaction, robot assisted therapy, humanoids and rehabilitation robotics with core technology of using variable impedance actuators.

W. H. Zhu is an engineering technical officer at the Canadian Space Agency. His specialty is on precision control of complex robotic systems characterized by his book entitled Virtual Decomposition Control published by Springer-Verlag in its STAR series. Dr. Zhu also published 60+ papers in leading international journals and conference proceedings. 